TRANSACTIONS OF THE

AMERICAN MATHEMATICAL SOCIETY

Volume 354, Number 2, Pages 735-747

S 0002-9947(01)02889-6

Article electronically published on October 3, 2001

\title{
THE A-POLYNOMIAL FROM THE NONCOMMUTATIVE VIEWPOINT
}

\author{
CHARLES FROHMAN, RĂZVAN GELCA, AND WALTER LOFARO
}

\begin{abstract}
The paper introduces a noncommutative generalization of the Apolynomial of a knot. This is done using the Kauffman bracket skein module of the knot complement, and is based on the relationship between skein modules and character varieties. The construction is possible because the Kauffman bracket skein algebra of the cylinder over the torus is a subalgebra of the noncommutative torus. The generalized version of the A-polynomial, called the noncommutative A-ideal, consists of a finitely generated ideal of polynomials in the quantum plane. Some properties of the noncommutative A-ideal and its relationships with the A-polynomial and the Jones polynomial are discussed. The paper concludes with the description of the examples of the unknot, and the right- and left-handed trefoil knots.
\end{abstract}

\section{INTRODUCTION}

This paper places the A-polynomial of a knot into the framework of noncommutative geometry. The A-polynomial was introduced in [CCGLS]. It describes how the $\mathrm{Sl}_{2} \mathbb{C}$-characters of a knot lie inside the $S l_{2} \mathbb{C}$-characters of its boundary torus, and can be related to the Alexander polynomial of the knot and to the structure of essential surfaces in the complement of the knot [CL].

The noncommutative generalization is constructed by replacing the geometric definition of the A-polynomial by the algebraic definition based on spaces of functions, and then deforming these spaces with respect to a complex parameter $t$. As such, $\mathrm{Sl}_{2} \mathbb{C}$-characters of surfaces and 3-manifolds are replaced by Kauffman bracket skein modules of cylinders over surfaces and 3-manifolds. The Kauffman bracket skein module depends on a parameter $t$, so that when $t$ is set equal to -1 the $S l_{2} \mathbb{C}$-characters are recovered. The A-polynomial can be derived from the noncommutative invariant at $t=-1$.

Our construction uses the isomorphism between the Kauffman bracket skein module of the cylinder over a torus and the subalgebra of the noncommutative torus generated by noncommutative cosines, which was already explicated in [FG]. The noncommutative torus is a fundamental example in noncommutative geometry Co.

The invariant is a left ideal of polynomials in the noncommutative plane. Since the noncommutative plane is a Noetherian algebra which admits a Gröbner basis algorithm for the lexical order, this ideal is finitely generated and admits a minimal

Received by the editors March 14, 2001 and, in revised form, May 7, 2001.

1991 Mathematics Subject Classification. Primary 57M25, 58B30, 46L87.

Key words and phrases. Kauffman bracket, skein modules, A-polynomial, character varieties, noncommutative geometry. 
reduced Gröbner basis. The use of Gröbner bases is an important theme in modern commutative algebra. For instance, algorithms associated with Gröbner bases are the heart of most symbolic manipulation programs. The use of Gröbner bases to study the noncommutative plane is foreshadowed by the work of Cohn on skew polynomial rings $\mathrm{C}$.

There is a complex valued pairing induced by the tensor product of the skein module of the knot complement with the skein module of the solid torus over the skein algebra of the boundary torus. Topologically the pairing is determined by the gluing of the solid torus to the knot complement. This allows us to compare the noncommutative invariant with data coming from colored Jones polynomials. Specifically, the matrix associated to the noncommutative invariant annihilates the vector whose entries are the colored Jones polynomials. This should lead to a deeper understanding of the relationship between the Jones polynomial and the representation theory of the fundamental group of the complement of a knot.

In section 2 the basic definitions associated with the A-polynomial are recalled. In section 3 we introduce the noncommutative analogues of the spaces and maps used in section 2. In section 4 we define the noncommutative A-ideal, describe some of its properties and discuss the examples of the unknot and the left- and right-handed trefoils. In section 5 we derive the orthogonality relationship between the noncommutative A-ideal and the Jones polynomial.

\section{Characters And Knot invariants}

2.1. $S l_{2} \mathbb{C}$-Characters. Let $G=\left\langle a_{i} \mid r_{j}\right\rangle$ be a finitely generated group. A representation $\rho: G \rightarrow S l_{2} \mathbb{C}$ is determined by a choice of matrices $A_{i}$ in $S l_{2} \mathbb{C}$ so that when the relations $r_{j}$ are rewritten with the $a_{i}$ replaced with the $A_{i}$ they are equal to the identity in $S l_{2} \mathbb{C}$. In other words, the representations can be identified with the subset of $\prod_{i=1}^{n} S l_{2} \mathbb{C}$, where $n$ is the number of generators of $G$, that satisfies the equations obtained by requiring the $r_{j}$ to evaluate to the identity. Denote this subset $\operatorname{Rep}(G)$, and call it the representations of $G$. Since we only deal with $\mathrm{Sl}_{2} \mathbb{C}$-representations, we suppress references to $\mathrm{Sl}_{2} \mathbb{C}$.

Let $a_{r s}(i), r, s \in\{1,2\}$, and $i \in\{1, \ldots, n\}$ be the function on $\prod_{i=1}^{n} S l_{2} \mathbb{C}$ which yields the entry in the $r$-th row and $s$-th column of the $i$-th matrix of each element of $\prod_{i=1}^{n} S l_{2} \mathbb{C}$. The coordinate ring $C\left[\prod_{i=1}^{n} S l_{2} \mathbb{C}\right]$ consists of the polynomials in the $a_{r s}(i)$ modulo the ideal generated by

$$
a_{11}(i) a_{22}(i)-a_{12}(i) a_{21}(i)-1,
$$

for $i \in\{1, \ldots, n\}$. Each relation $r_{j}$ gives rise to four polynomials corresponding to the four entries of a matrix, and coming from the condition that the relations evaluate to the identity. Let $I(G)$ be the ideal in $C\left[\prod_{i=1}^{n} S l_{2} \mathbb{C}\right]$ generated by the polynomials coming from the relations. Finally, $\mathcal{R}(G)=C\left[\prod_{i=1}^{n} S l_{2} \mathbb{C}\right] / I(G)$ is the affine representation ring of $G$. In more generality, Lubotsky and Magid [LM] proved that the isomorphism class of $\mathcal{R}(G)$ is an invariant of the group. The word "affine" refers to the fact that $\mathcal{R}(G)$ is the unreduced coordinate ring of the representations. To get the classical representation ring, take the quotient of $\mathcal{R}(G)$ by its nilradical $\sqrt{0}=\left\{p \mid p^{n}=0\right.$ for some $\left.n\right\}$.

There is a left action of $S l_{2} \mathbb{C}$ on $\prod_{i=1}^{n} S l_{2} \mathbb{C}$ by conjugation,

$$
A \bullet\left(A_{1}, \ldots, A_{n}\right)=\left(A A_{1} A^{-1}, \ldots, A A_{n} A^{-1}\right) .
$$


This action induces a right action on $C\left[\prod_{i=1}^{n} S l_{2} \mathbb{C}\right]$. It is easy to check that the action leaves $I(G)$ invariant, hence the action descends to a right action on $\mathcal{R}(G)$. The invariant subring of this action, denoted $\chi(G)=\mathcal{R}(G)^{S l_{2} \mathbb{C}}$, is called the affine characters of $G$. This ring is an invariant of the group $G$. Once again, to obtain what is classically referred to as the characters, take the quotient of $\chi(G)$ by its nilradical.

For the most frequently encountered groups we are being too careful, the affine representation ring and the affine character ring have trivial nilradical. However, there are examples of groups where the distinction is real [KM].

The affine characters have recently been the subject of scrutiny in works of Bullock, Brumfiel-Hilden, Przytycki-Sikora, and Sikora, [B, BH, PS, S1. Here is an intrinsic definition due to Sikora. Let $\hat{G}$ be the set of conjugacy classes of $G$. If $W$ is an element of $G$ we denote the conjugacy class of $W$ by $\langle W\rangle$. Let $S(\hat{G})$ be the symmetric algebra on $\hat{G}$, that is, polynomials where the variables are conjugacy classes in $G$ and the coefficients are complex numbers. Let $J$ be the ideal generated by all polynomials, $\langle I d\rangle+2$, and $\langle A B\rangle+\left\langle A^{-1} B\right\rangle+\langle A\rangle\langle B\rangle$ where $A$ and $B$ range over the elements of $G$. An isomorphism $S(G) / J \rightarrow \chi(G)$ is induced by sending each $\langle A\rangle$ into the polynomial corresponding to $-\operatorname{tr}(A)$.

There is a space corresponding to the characters. The action of $\mathrm{Sl}_{2} \mathbb{C}$ on the ring $C\left[\prod_{i=1}^{n} S l_{2} \mathbb{C}\right]$ is not good, in the sense that the quotient is not Hausdorff. We sidestep this by defining an equivalence relation on $\operatorname{Rep}(G)$, that yields a Hausdorff space. Let $\rho, \eta \in \operatorname{Rep}(G)$ be equivalent if for every $t \in \chi(G), t(\rho)=t(\eta)$. The resulting quotient space is the character variety of $G$. An algebraic subset of a $\mathbb{C}^{n}$ is the solution set of a system of polynomial equations. A set of polynomials $S$ cuts out an algebraic subset $V$, if $x \in V$ if and only if every function in $S$ is zero at $x$. The ideal $I(V)$ of $V$ is the set of all polynomials that vanish on $V$. It is a consequence of the Nullstellensatz that the ideal of $V$ is the radical of the smallest ideal containing any set of polynomials that cut $V$ out. The coordinate ring of $V$ is the quotient of the ring of polynomials in $n$ variables by $I(V)$. Sometimes it is nice to have a set $X(G)$ corresponding to the characters $\chi(G)$. Of course the set only matters to the extent that its points correspond to maximal ideals in $\chi(G)$. To this end, define a realization of $X(G)$ to be an algebraic subset of some $\mathbb{C}^{n}$, where the coordinates on $\mathbb{C}^{n}$ correspond to traces of elements of $G$, whose coordinate ring is isomorphic to $\chi(G) / \sqrt{0}$. The set $X(G)$ is in one to one correspondence with the character variety of $G[\mathrm{CS}]$.

2.2. Characters of the Torus. Concentrate on the case of the fundamental group of a torus. Think of it as the free Abelian group on $\lambda$ and $\mu$. The representations correspond exactly to pairs of matrices in $S l_{2} \mathbb{C}$ that commute. Let $x$ be the trace of the image of $\lambda, y$ be the trace of the image of $\mu$ and let $z$ be the trace of the image of their product. The affine character ring, $\chi\left(\pi_{1}\left(T^{2}\right)\right)$, is generated by $x, y$, $z$ with relation

$$
x^{2}+y^{2}+z^{2}-x y z-4=0 .
$$

The ring has trivial nilradical. The space $X\left(T^{2}\right)=X\left(\pi_{1}\left(T^{2}\right)\right)$ is realized as the set of points in $\mathbb{C}^{3}$ satisfying the equation above. There is a two-fold branched cover of $X\left(T^{2}\right)$ that is used in the definition of knot invariants. Consider $\mathbb{C}^{*} \times \mathbb{C}^{*}$, where $\mathbb{C}^{*}$ denotes the nonzero complex numbers, give it coordinates $l$ and $m$. In order to see that its coordinate ring is $\mathbb{C}\left[l, l^{-1}, m, m^{-1}\right]$, it is helpful to think of $\mathbb{C}^{*} \times \mathbb{C}^{*}$ as 
ordered pairs of diagonal matrices in $\mathrm{Sl}_{2} \mathbb{C}$ :

$$
\left(\left(\begin{array}{cc}
l & 0 \\
0 & l^{-1}
\end{array}\right),\left(\begin{array}{cc}
m & 0 \\
0 & m^{-1}
\end{array}\right)\right) .
$$

There is a map $p: \mathbb{C}^{*} \times \mathbb{C}^{*} \rightarrow X\left(T^{2}\right)$ given by sending each pair of points to its equivalence class. The map $p$ is a two-fold branched cover whose singular points are the four ordered pairs chosen from $\left(\begin{array}{cc} \pm 1 & 0 \\ 0 & \pm 1\end{array}\right)$. In terms of our realization of $X\left(T^{2}\right)$, the map sends

$$
\left(\left(\begin{array}{cc}
l & 0 \\
0 & l^{-1}
\end{array}\right),\left(\begin{array}{cc}
m & 0 \\
0 & m^{-1}
\end{array}\right)\right)
$$

to the triple $\left(l+l^{-1}, m+m^{-1}, l m+l^{-1} m^{-1}\right)$. Being a two-fold branched cover, $p$ has a deck transformation,

$$
\theta: \mathbb{C}^{*} \times \mathbb{C}^{*} \rightarrow \mathbb{C}^{*} \times \mathbb{C}^{*}
$$

$\theta(l, m)=\left(l^{-1}, m^{-1}\right)$.

Dual to $p$, and $\theta$ are maps, that we denote $\hat{p}$ and $\hat{\theta}$,

$$
\hat{p}: C\left[X\left(T^{2}\right)\right] \rightarrow \mathbb{C}\left[l, l^{-1}, m, m^{-1}\right],
$$

and

$$
\hat{\theta}: \mathbb{C}\left[l, l^{-1}, m, m^{-1}\right] \rightarrow \mathbb{C}\left[l, l^{-1}, m, m^{-1}\right] .
$$

One can show that the image of $\hat{p}$ is the fixed subalgebra of $\hat{\theta}$.

Proposition 1. Suppose that $V \subset X\left(T^{2}\right)$ is algebraic and $S$ is a set of functions that cuts out $V$. The ideal of $p^{-1}(V)$ is the radical of the smallest ideal of $\mathbb{C}\left[l, l^{-1}, m, m^{-1}\right]$ containing $\hat{p}(S)$.

Proof. The set $\hat{p}(S)$ cuts out $p^{-1}(V)$, the rest is a standard characterization of the ideal of an algebraic set.

The coordinate ring $\mathbb{C}\left[l, l^{-1}, m, m^{-1}\right]$ can be seen as the ring of fractions of $\mathbb{C}[l, m]$ with respect to the set of monomials in $l$ and $m$.

Proposition 2. If $I \subset \mathbb{C}\left[l, l^{-1}, m, m^{-1}\right]$ is an ideal, then its contraction $J$ to $\mathbb{C}[l, m]$ is $I \cap \mathbb{C}[l, m]$. The extension of $J$ is just the ideal of $\mathbb{C}\left[l, l^{-1}, m, m^{-1}\right]$ generated by $J$. Furthermore, the extension of $J$ is $I$.

2.3. The A-polynomial. The A-polynomial is defined in CCGLS. Here is a brief overview of that definition. Let $K$ be a knot in $S^{3}$, or more generally in an oriented homology 3 -sphere. Let $M$ be the complement of a regular neighborhood of $K$. The space $M$ is a compact manifold with boundary homeomorphic to $T^{2}$. To choose the meridian and the longitude of the knot, orient the knot and choose the meridian to bound a disk in the regular neighborhood and to have the linking number with the knot equal to +1 . The longitude bounds a Seifert surface and has intersection number with the meridian +1 , thus we choose the positive orientation on the boundary torus. This determines the longitude and the meridian up to sign. The inclusion map $T^{2}=\partial M \subset M$ induces a map $r: X(M) \rightarrow X\left(T^{2}\right)$. Consider the closure of the 1-dimensional part of the peripheral characters $r(X(M))$. Its pull-back through the map $\left.p: \mathbb{C}^{*} \times \mathbb{C}^{*} \rightarrow X\left(T^{2}\right)\right)$ is still 1-dimensional since $p$ is surjective and is $2-1$ except at four points. Viewed as a curve in $\mathbb{C} \times \mathbb{C}$, this set is the zero set of a two-variable polynomial $B(l, m)$, which is unique if we require that 
all of its factors are simple and that it is monic. This polynomial always factors as $B(l, m)=(l-1) A(l, m)$, where the factor $l-1$ stands for the curve of characters of reducible representations. The factor $A(l, m)$ is by definition the A-polynomial of $K$.

\section{Skein MOdules AND THE NONCOMmUtATive torus}

3.1. The Noncommutative Torus. The noncommutative torus was first introduced by Rieffel [Ri1] as an example in the study of noncommutative $C^{*}$-algebras, its physical significance and geometric properties were only later discovered [C], [Ri2], [Ri3], [We]. The noncommutative torus is a "virtual" geometric space whose algebra of continuous functions is the (irrational) rotation algebra $A_{\theta}$. It is customary to call the algebra of functions itself the noncommutative torus.

The algebra $A_{\theta}$ is usually defined for a real angle of rotation $\theta$, however we con$\operatorname{sider} \theta$ to be any complex number, and let $t=e^{\pi i \theta}$. This algebra can be introduced abstractly by exponentiating the Heisenberg noncommutation relation. That is, $A_{\theta}$ is the closure in a certain $C^{*}$-norm of the algebra spanned by $l, m, l^{-1}, m^{-1}$, subject to the relation $l m=t^{2} m l$.

One can also define $A_{\theta}$ by its multiplication rule. Consider the elements $e_{p, q}=$ $t^{-p q} l^{p} m^{q}, p, q \in \mathbb{Z}$. They span over $\mathbb{C}$ a dense subalgebra. Define the multiplication via the formula

$$
e_{p, q} * e_{r, s}=t^{p q} \mid e_{p+r, q+s}
$$

which, from our approach, is just a consequence of the defining relation. The irrational rotation algebra is the closure of the algebra spanned by the $e_{p, q}$ 's in the norm determined by the left regular representation (with this new product) on $L^{2}\left(T^{2}\right)$.

For the purpose of this paper we are interested only in the subalgebra of the noncommutative torus consisting of Laurent polynomials in $l$ and $m$, which we denote by $\mathbb{C}_{t}\left[l, l^{-1}, m, m^{-1}\right]$. There is an automorphism

$$
\Theta: \mathbb{C}_{t}\left[l, l^{-1}, m, m^{-1}\right] \rightarrow \mathbb{C}_{t}\left[l, l^{-1}, m, m^{-1}\right], \quad \Theta\left(e_{p, q}\right)=e_{-p,-q} .
$$

Let $\mathcal{C}_{t}$ be its invariant part. The notation is motivated by the fact that this subalgebra is spanned by the noncommutative cosines $\frac{1}{2}\left(e_{p, q}+e_{-p,-q}\right), p, q \in \mathbb{Z}$.

In addition, let $\mathbb{C}_{t}[l, m]$ be the subalgebra of $\mathbb{C}_{t}\left[l, l^{-1}, m, m^{-1}\right]$ spanned by $e_{p, q}$, with $p, q \geq 0$. This is nothing but the ring of noncommuting polynomials in two variables $l$ and $m$ satisfying the noncommutation relation $l m=t^{2} m l$. This ring is frequently referred to as the quantum plane.

Proposition 3 ([ $[\mathrm{K}]$, Proposition IV.1.1). The ring $\mathbb{C}_{t}[l, m]$ is both left and right Noetherian and has no zero divisors.

We now need to broach the subject of Gröbner bases in $\mathbb{C}_{t}[l, m]$. As $\mathbb{C}_{t}[l, m]$ is so close to being commutative, the concepts translate over very easily from the case of two-variable polynomials.

We lexicographically order the $l^{p} m^{q}$. Hence $l^{p} m^{q}<l^{r} m^{s}$ if either $p<r$ or $p=r$ and $q<s$. Given $f \in \mathbb{C}_{t}[l, m]$ we can write $f=\sum \alpha_{p, q} e_{p, q}$ where the sum is finite. The leading term $l t(f)$ of $f$ is the $\alpha_{p, q} l^{p} m^{q}$ where the $l^{p} m^{q}$ is largest in the lexicographical ordering among those terms with $\alpha_{p, q} \neq 0$. The leading power product is $l^{p} m^{q}$ and the leading coefficient is $\alpha_{p, q}$. 
Suppose that $u, v, w \in \mathbb{C}_{t}[l, m]$ and $u=v w$, then we say $w$ divides $u$ on the right and we let $\frac{u}{w}=v$. A Gröbner basis for a left (respectively right) ideal $I$ is a collection $f_{i}$ of elements of the ideal $I$ so that the ideal generated by the leading terms of the $f_{i}$ is equal to the ideal generated by the leading terms of elements of $I$. We say the Gröbner basis is minimal if no two $f_{i}$ have the same leading power product. We say the Gröbner basis is reduced if no power product in each $f_{i}$ is divisible by the leading power product of any other $f_{i}$.

Proposition 4. For a left (or right) ideal of polynomials in $\mathbb{C}_{t}[l, m]$ there exists a unique minimal, reduced Gröbner basis, consisting of monic polynomials.

Proof. By changing any statements in $\mathrm{AL}$ about ideals to statements about left (or right) ideals, the proof there goes through verbatim.

3.2. The Kauffman Bracket Skein Module. Let $M$ be an orientable 3-manifold. A framed link in $M$ is an embedding of a disjoint union of annuli into $M$. In diagrams we will draw only the core of an annulus lying parallel to the plane of the paper (i.e. with blackboard framing).

Two framed links in $M$ are equivalent if there is an isotopy of $M$ taking one to the other. Let $\mathcal{L}$ denote the set of equivalence classes of framed links in $M$, including the empty link. Fix a complex number $t$. Consider the vector space, $\mathbb{C} \mathcal{L}$ with basis $\mathcal{L}$. Define $S(M)$ to be the smallest subspace of $\mathbb{C} \mathcal{L}$ containing all expressions of the form

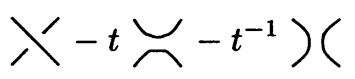

and $\bigcirc+t^{2}+t^{-2}$, where the framed links in each expression are identical outside balls, in which they look as pictured in the diagrams. The Kauffman bracket skein module $K_{t}(M)$ is the quotient

$$
\mathbb{C} \mathcal{L} / S(M) .
$$

In the case of the cylinder over the torus, $T^{2} \times I, K_{t}\left(T^{2} \times I\right)$ has the structure of an algebra with multiplication given by laying one link over the other. More precisely, to multiply skeins corresponding to links $\alpha$ and $\beta$, isotope them so that $\alpha$ lies in $T^{2} \times\left(\frac{1}{2}, 1\right]$ and $\beta$ in $T^{2} \times\left[0, \frac{1}{2}\right)$. Then $\alpha \cdot \beta$ is the element of the skein module represented by the class of the union of these two links in $T^{2} \times[0,1]$. Extend this to a distributive product.

Oriented simple closed curves on the torus up to isotopy are indexed by pairs of relatively prime integers $(p, q)$. Corresponding to $(p, q)$ is a framed link in $T^{2} \times I$. Take an annulus in $T^{2} \times I$ whose core projects to a $(p, q)$ curve, so that the annulus runs parallel to the boundary of $T^{2} \times I$. As the framed links are unoriented, $(p, q)$ and $(-p,-q)$ give rise to the same link, which we also denote by $(p, q)$. A standard argument based on the proof that the Kauffman bracket in $S^{3}$ is well defined shows that as a vector space, $K_{t}\left(T^{2} \times I\right)$ has as basis all links consisting of parallel copies of the $(p, q)$.

Let $x$ be $(0,1), y=(1,0)$ and $z=(1,1)$. It is a theorem of Bullock and Przytycki $[\mathrm{BP}]$ that $K_{t}\left(T^{2} \times I\right)$ is isomorphic to polynomials in three noncommutative variables, $x, y$ and $z$ modulo the ideal generated by

$$
\begin{gathered}
t^{2} x^{2}+t^{-2} y^{2}+t^{2} z^{2}-t x y z-2\left(t^{2}+t^{-2}\right), \\
t x y-t^{-1} y x-\left(t^{2}-t^{-2}\right) z
\end{gathered}
$$




$$
t z x-t^{-1} x z-\left(t^{2}-t^{-2}\right) y
$$

and

$$
t y z-t^{-1} z y-\left(t^{2}-t^{-2}\right) x .
$$

When $t=-1$ the Kauffman bracket skein module of an arbitrary three-manifold can be made into an algebra. The point is that at $t=-1$ the skein relation allows us to change crossings. To multiply two links, perturb them so that they miss one another and take their union. As crossings don't count, the answer is independent of the perturbation. This extends to make $K_{-1}(M)$ into an algebra for any $M$. It is a theorem of Bullock $[\mathrm{B}]$ that $K_{-1}(M) / \sqrt{0}$ is naturally isomorphic to $\chi\left(\pi_{1}(M)\right) / \sqrt{0}$. You can see this isomorphism from the description of $\chi\left(\pi_{1}(M)\right)$ due to Sikora given above. The correspondence at $t=-1$ is slightly tricky as the $x, y$ and $z$ given here correspond to $-x,-y$ and $-z$ in the relation we gave for the $S L_{2} \mathbb{C}$-characters of the torus.

In $[\mathrm{FG}$ ] the following theorem is proved.

Theorem 1. There exists an isomorphism of algebras

$$
\hat{p}: K_{t}\left(T^{2} \times I\right) \rightarrow \mathcal{C}_{t}
$$

determined by

$$
\hat{p}((p, q))=e_{p, q}+e_{-p,-q}, \quad p, q \in \mathbb{Z}
$$

\section{The noncommutative A-IDEAL}

4.1. Definition. In this section we introduce a noncommutative generalization of the A-polynomial, depending on the complex parameter $t$, from which the Apolynomial can be recovered by letting $t=-1$. To define this new knot invariant, we replace the geometric definition of the A-polynomial, based on the character variety, by a definition based on the space of regular functions on the character variety, then deform this space of functions with respect to a parameter. Through this procedure, the algebra of functions on the character variety of the torus deforms to a noncommutative algebra, while the algebra of functions on the knot complement deforms to a right module over the algebra of the torus.

Denote by $\mathcal{I}(K)$ the kernel of

$$
\hat{r}: \chi\left(\pi_{1}\left(T^{2}\right)\right) \rightarrow \chi\left(\pi_{1}\left(S^{3}-K\right)\right) .
$$

Notice that $\mathcal{I}(K)$ cuts out an algebraic subset $V$ of $X\left(T^{2}\right)$.

Proposition 5. Let $J$ be the ideal generated by $\hat{p}(\mathcal{I}(K))$ in $\mathbb{C}\left[l, l^{-1}, m, m^{-1}\right]$, and $J^{c}$ its contraction to $\mathbb{C}[l, m]$. Then the product of the generators of the principal minimal prime ideals associated to $J^{c}$ is equal to $B(l, m)$ (modulo multiplication by a unit). That is, the ideal $\mathcal{I}(K)$ determines the A-polynomial.

Proof. The ideal $J$ cuts out the preimage in $\mathbb{C}^{*} \times \mathbb{C}^{*}$ through the map $p$, of the variety of peripheral characters, and consequently $J^{c}$ cuts out this variety as viewed in $\mathbb{C} \times \mathbb{C}$. By restricting ourselves to the 1-dimensional part, the conclusion follows.

You can think of the extension of the ideal $\mathcal{I}(K)$ as the holomorphic sections of a line bundle over $\mathbb{C}^{*} \times \mathbb{C}^{*}$, specifically, the sections of the line bundle associated to the divisor of $\frac{1}{B(l, m)}$. 
There is a map $\hat{r}: K_{t}\left(T^{2} \times I\right) \rightarrow K_{t}\left(S^{3}-K\right)$ obtained by gluing the cylinder over a torus into the complement of the knot at the $T^{2} \times\{0\}$ end so that the meridian goes to the meridian and the longitude goes to the longitude. Let $\mathcal{I}_{t}(K)$ be the kernel of $\hat{r}$. This ideal is called the peripheral ideal of the knot. Recall that for any 3-manifold $K_{-1}(M) / \sqrt{0}$ is isomorphic to $\chi\left(\pi_{1}(M)\right) / \sqrt{0}$. For a cylinder over a torus the radical of both rings is trivial, hence

$$
K_{-1}\left(T^{2} \times I\right)=\chi\left(\pi_{1}\left(T^{2}\right)\right)=C\left(X\left(T^{2}\right)\right) .
$$

Under this identification $\mathcal{I}_{-1}(K)=\mathcal{I}(K)$. If we extend the ideal $\mathcal{I}_{-1}(K)$ to $K_{-1}\left(\mathbb{C}^{*} \times \mathbb{C}^{*}\right)=\mathbb{C}_{-1}\left[l, l^{-1}, m, m^{-1}\right]$ and then contract it to $K_{-1}(\mathbb{C} \times \mathbb{C})=\mathbb{C}_{-1}[l, m]$, we get an ideal of polynomials in two variables. From the above proposition it follows that the principal minimal primes associated to this ideal are generated by polynomials whose product is $B(-l,-m)$ (eventually multiplied by a unit). The negatives are due to the fact that the skein relation corresponds to the identity satisfied by the negative of the trace.

This motivates the following

Definition 1. The noncommutative A-ideal $\mathcal{A}_{t}(K)$ of a knot $K$ is the left ideal obtained by extending $\mathcal{I}_{t}(K)$ to $\mathbb{C}_{t}\left[l, l^{-1}, m, m^{-1}\right]$ and then contracting it to $\mathbb{C}_{t}[l, m]$.

\subsection{Properties.}

Proposition 6. The noncommutative A-ideal at $t=-1$ determines the A-polynomial.

Proof. This follows immediately from Proposition 5.

Lemma 1. The left ideal obtained by extending a left ideal $\mathcal{I} \in K_{t}\left(T^{2} \times I\right)$ to the algebra $\mathbb{C}_{t}\left[l, l^{-1}, m, m^{-1}\right]$ and then contracting it back to $K_{t}\left(T^{2} \times I\right)$ coincides with $\mathcal{I}$.

Proof. The algebra $K_{t}\left(T^{2} \times I\right)$ consists of those Laurent polynomials in $l$ and $m$ that are invariant under the transformation $\Theta$ defined by $l \rightarrow l^{-1}$ and $m \rightarrow m^{-1}$. If $r(l, m)$ is an element in the ideal obtained through the extension followed by the contraction, then $r(l, m)$ is invariant under $\Theta$ and

$$
r(l, m)=\sum_{j} p_{j}(l, m) q_{j}(l, m)
$$

where $q_{j} \in \mathcal{I}$ for all $j$. If we symmetrize this equality with respect to the transformation $\Theta$, then we get

$$
r(l, m)=\sum_{j} \frac{1}{2}\left(p_{j}(l, m)+p_{j}\left(l^{-1}, m^{-1}\right)\right) q_{j}(l, m) .
$$

The polynomials in the brackets are now invariant under $\Theta$, which shows that $r(l, m) \in \mathcal{I}$, and we are done.

Proposition 7. The peripheral ideals of two knots are the same if and only if their noncommutative $A$-ideals are the same.

Proof. From the definition of the noncommutative A-ideal it follows that $I_{t}(K)$ determines $\mathcal{A}_{t}(K)$. The converse follows from the above lemma and the fact that the operations of extension and contraction between the ideals of $\mathbb{C}_{t}[l, m]$ and those of $\mathbb{C}_{t}\left[l, l^{-1}, m, m^{-1}\right]$ are inverses of each other. 
Proposition 8. The noncommutative A-ideal is nontrivial.

Proof. We saw in the proof of the previous proposition that the peripheral ideal $\mathcal{I}_{t}(K)$ can be recovered by extending $\mathcal{A}_{t}(K)$ to $\mathbb{C}_{t}\left[l, l^{-1}, m, m^{-1}\right]$ and then contracting it to the Kauffman bracket skein algebra of the torus. Hence the noncommutative A-ideal is nontrivial if and only if the peripheral ideal is nontrivial. To prove that the peripheral ideal is nontrivial consider the map $r: K_{t}\left(T^{2} \times I\right) \rightarrow K_{t}\left(S^{3} \backslash K\right)$. As a submodule, $I_{t}(K)$ is the kernel of this map. If $I_{t}(K)=(0)$ then $r$ is an isomorphism onto the image.

If we add a 2-handle to the knot complement along the meridian of the knot, then the skein module of the knot complement, and hence the image of $r$ becomes $\mathbb{C}$. If we add a 2-handle to the cylinder over the torus along the meridian, the skein module becomes $\mathbb{C}[\alpha]$, where $\alpha$ is the longitude, and this is an infinite dimensional space. But Hoste and Przytycki HP] have shown that adding a 2-handle induces a set of local algebraic relations at the level of the skein module, and assuming $r$ to be an isomorphism, the same relations are introduced in $K_{t}\left(\mathbb{T}^{2} \times I\right)$ and in its image through $r$. Since adding the same relations produces different modules, $r$ is not an isomorphism, therefore it has a kernel.

Proposition 9. The noncommutative A-ideal is generated by finitely many polynomials with coefficients in $\mathbb{Z}\left[t, t^{-1}\right]$.

Proof. The computation of generators for the peripheral ideal reduces to the repeated use of the Kauffman bracket skein relation in the knot complement. Hence the peripheral ideal and thus the noncommutative A-ideal is generated by polynomials with coefficients in $\mathbb{Z}\left[t, t^{-1}\right]$. The Noetherianity of the quantum plane implies that the noncommutative A-ideal is generated by finitely many such polynomials.

Note that by using the Gauss lemma in the case $t=-1$ we recover the well known fact that the A-polynomial has integer coefficients.

The theory of Kauffman bracket skein modules has been developed assuming $t$ to be a variable rather than a complex number. To avoid confusion we use the notation $\tau$ instead of $t$ for the variable (the established notation is $A$, which is inappropriate here). All the consideration in the paper apply mutatis mutandis to define a knot invariant $\mathcal{A}_{\tau}(K)$ which is a finitely generated ideal in $\mathbb{C}\left[\tau, \tau^{-1}\right][l, m]$.

Proposition 10. The ideal $\mathcal{A}_{t}(K)$ includes the ideal obtained from $\mathcal{A}_{\tau}(K)$ by specializing $\tau=t$. If $t$ is transcendental this inclusion is an equality.

Proof. The skein algebra $K_{t}\left(T^{2} \times I\right)$ is obtained from $K_{\tau}\left(T^{2} \times I\right)$ by specializing $\tau=t$. The image of an element from the peripheral ideal $\mathcal{I}_{\tau}(K)$ through the inclusion of the boundary torus into the knot complement becomes zero after finitely many applications of the Kauffman bracket skein relation. Applying the same skein relations after specializing $\tau=t$ shows that this element gives rise to an element in $\mathcal{I}_{t}(K)$. This proves the inclusion.

Since finding generators for $\mathcal{I}_{t}(K)$ (respectively $\mathcal{I}_{\tau}(K)$ ) amounts to using the Kauffman bracket skein relation finitely many times, the computation of these generators can be done assuming that the ring of coefficients is $\mathbb{Q}\left[t, t^{-1}\right]$ (respectively $\mathbb{Q}\left[\tau, \tau^{-1}\right]$ ) instead of $\mathbb{C}\left[t, t^{-1}\right]$ (respectively $\mathbb{C}\left[t, t^{-1}\right]$ ). For a particular $t$ assume that the inclusion is strict. Then there exists an element $\gamma \in \mathcal{I}_{t}(K)$ that is not obtained from $\mathcal{I}_{\tau}(K)$ by specializing the variable. Then $\gamma$ arises through the specialization 
from an element in $K_{\tau}\left(T^{2} \times I\right)$ of the form $\gamma_{0}+p(\tau) \gamma_{1}$, where $\gamma_{0} \in \mathcal{I}_{\tau}(K)$ and $p$ is a Laurent polynomial divisible by $\tau-t$. By multiplying with the appropriate power of $\tau$ we can transform $p$ into a polynomial still divisible by $\tau-t$. It follows that $t$ is the root of a polynomial with rational coefficients, hence it is algebraic.

The computation done in the case of the trefoil knot suggests that the above inclusion is strict only for finitely many values of $t$.

4.3. Examples. 1. $K$ is the unknot. From $[\mathrm{FG}$ we get that the peripheral ideal $\mathcal{I}_{t}(K)$ is generated by $(1,0)+t^{2}+t^{-2}$ and $(1,1)+t^{3}(0,1)$. The noncommutative A-ideal is generated by $\left(l+t^{2}\right)\left(l+t^{-2}\right)$ and $l m^{2}\left(l+t^{2}\right)+t^{2}\left(l+t^{-2}\right)$. An easy application of Buchberger's Algorithm yields the minimal reduced Gröbner basis $\left\{l^{2}+\left(t^{2}+t^{-2}\right) l+1, l m^{2}-t^{4} l+t^{6} m^{2}-t^{2}\right\} .\left(l+t^{2}\right)\left(l+t^{-2}\right)$ For $t=-1$, the ideal is generated by $(l+1)^{2}$ and $\left(m^{2}+1\right)(l+1)$. To get the classical A-polynomial take the radical and get the ideal generated by $B(-l,-m)=l+1$. Thus $B(l, m)=l-1$ and $A(l, m)=1$ which is what we expected to get. Note that in addition to the Apolynomial, the A-ideal detects the embedded primes corresponding to the points $(1, i)$ and $(1,-i)$.

2. $K$ is the right-handed trefoil. It was shown in $\mathrm{Ge}$ that for $t$ not an eighth root of unity, the noncommutative A-ideal is generated by the polynomials

$$
\begin{aligned}
& {\left[m^{4}\left(l+t^{10}\right)-t^{-4}\left(l+t^{2}\right)\right]\left(l m^{6}-t^{6}\right),} \\
& \left(l+t^{24}\right)\left(l+t^{10}\right)\left(l+t^{2}\right)\left(l m^{6}-t^{6}\right), \\
& \left(m^{2}-t^{-22}\right)\left(l+t^{10}\right)\left(l+t^{2}\right)\left(l m^{6}-t^{6}\right) .
\end{aligned}
$$

A minimal reduced Gröbner basis for this is

$$
\begin{aligned}
& t^{20}-t^{32} m^{4}+t^{18} l-t^{14} l m^{4}-t^{14} l m^{6}+t^{18} l m^{10}-t^{12} l^{2} m^{6}+l^{2} m^{10}, \\
& -t^{18}-\left(t^{16}+t^{8}\right) l+t^{12} l m^{6}-t^{6} l^{2}+\left(t^{10}+t^{2}\right) l^{2} m^{6}+l^{3} m^{6} .
\end{aligned}
$$

One should note that in this case the peripheral ideal is generated by three elements while the noncommutative A-ideal is generated by only two.

The value $t=-1$ the A-ideal is generated by $\left(l^{2}-1\right)(l+1)\left(l m^{6}-1\right)$, and $\left(m^{2}-1\right)(l+1)\left(l m^{6}-1\right)$. The radical of the one-dimensional part of the ideal is generated by $(l+1)\left(l m^{6}-1\right)$. After changing $l$ to $-l$ and $m$ to $-m$ we get $B(l, m)=(l-1)\left(l m^{6}+1\right)$, that is $A(l, m)=\left(l m^{6}+1\right)$.

3. $K$ is the left-handed trefoil.

For $t$ not an eighth root of unity, the noncommutative A-ideal is generated by

$$
\begin{aligned}
& {\left[m^{4}\left(l+t^{10}\right)-t^{-4}\left(l+t^{2}\right)\right]\left(l-t^{6} m^{6}\right),} \\
& \left(l+t^{24}\right)\left(l+t^{10}\right)\left(l+t^{2}\right)\left(l-t^{6} m^{6}\right), \\
& \left(m^{2}-t^{-22}\right)\left(l+t^{10}\right)\left(l+t^{2}\right)\left(l-t^{6} m^{6}\right) .
\end{aligned}
$$

A minimal reduced Gröbner basis is

$$
\begin{array}{r}
t^{20} m^{6}-t^{32} m^{10}-t^{14} l+t^{18} l m^{4}+t^{18} l m^{6}-t^{14} l m^{10}-t^{12} l^{2}+l^{2} m^{4} \\
-t^{18} m^{6}+t^{34} m^{8}-t^{46} m^{12}+t^{12} l-t^{24} l m^{2}+\left(t^{28}-t^{16}-t^{8}\right) l m^{6} \\
+t^{28} l m^{8}-t^{24} l m^{12}+\left(t^{10}+t^{2}\right) l^{2}-t^{18} l^{2} m^{2}+l^{3}
\end{array}
$$

At $t=-1$ the ideal is generated by

$$
\left(l^{2}-1\right)(l+1)\left(l-m^{6}\right) m^{2} \text { and } \quad\left(m^{2}-1\right)(l+1)\left(l-m^{6}\right) .
$$

In this case one gets the classical A-polynomial $A(l, m)=l+m^{6}$. 


\section{Relation with the Jones POlynomial}

Assume in this section that $t$ is not a root of unity. The definition and normalization of the Jones-Wenzl idempotents will be used as in [Li]. Let $S_{c}$ be the skein in the solid torus obtained by plugging the $c$-th Jones-Wenzl idempotent into the core of the solid torus. The Kauffman bracket skein module of the solid torus has the set $\left\{S_{c}\right\}$ as a basis. Let $\hat{K}_{t}\left(S^{1} \times D^{2}\right)$ be the vector space of formal sums $\sum_{c} z_{c} S_{c}$ where the $z_{c}$ are complex numbers and the $c$ range over the natural numbers starting at 0 .

The double of the solid torus is $S^{1} \times S^{2}$. Any skein $\alpha$ in $S^{1} \times S^{2}$ can be represented by a linear combination of framed links that miss $1 \times S^{2}$. Hence, any skein in $S^{1} \times S^{2}$ can be represented as a skein in a punctured ball, and it has a Kauffman bracket. It follows that $K_{t}\left(S^{1} \times S^{2}\right)$ is canonically isomorphic to $\mathbb{C}$. There is a pairing between skeins in $K_{t}\left(S^{1} \times D^{2}\right)$. If $\alpha, \beta \in K_{t}\left(S^{1} \times D^{2}\right)$ are represented by a single framed link each, take the union of two copies of the solid torus, identified along their boundaries, with the link representing $\alpha$ in one and the link representing $\beta$ in the other. As this yields a skein in $S^{1} \times S^{2}$ we get a complex number by taking the Kauffman bracket as above. This can be extended bilinearly to give a pairing

$$
K_{t}\left(S^{1} \times D^{2}\right) \otimes \hat{K}_{t}\left(S^{1} \times D^{2}\right) \rightarrow \mathbb{C},
$$

for although the sum is infinite only finitely many terms are nonzero. In this way we identify $\hat{K}_{t}\left(S^{1} \times D^{2}\right)$ with $K_{t}\left(S^{1} \times D^{2}\right)^{*}$.

There is a representation of the Kauffman bracket skein algebra of the cylinder over the torus into endomorphisms of $\hat{K}_{t}\left(S^{1} \times D^{2}\right)$. Glue the cylinder onto $S^{1} \times D^{2}$ along the 0 -end of the cylinder so that longitudes and meridians go to longitudes and meridians. The matrix of a skein in the cylinder over the torus as a matrix with respect to the basis $S_{c}$, is of bounded width. That is, there is an integer $n$ so that if $|i-j|>n$ the $i j$-entry of the matrix is zero. This can be seen, as the matrix induced is the adjoint of the matrix corresponding to the endomorphism of $K_{t}\left(S^{1} \times D^{2}\right)$ induced by gluing the 1-end of the cylinder to $S^{1} \times D^{2}$. Notice that if $\hat{Z} \in \hat{K}_{t}\left(S^{1} \times D^{2}\right)$ then the annihilator of $\hat{Z}$ in the Kauffman bracket skein module of the cylinder over the torus is a left ideal.

Let $K \subset S^{3}$ be a framed knot. Let $X$ be the complement of an open regular neighborhood of the knot. There is a pairing

$$
K_{t}\left(S^{1} \times D^{2}\right) \otimes K_{t}(X) \rightarrow \mathbb{C}
$$

obtained by gluing the solid torus into the knot so that the meridian of the solid torus goes to the meridian of the knot and the blackboard longitude goes to the framing of the knot. To pair two skeins, take their union and then take the Kauffman bracket in $S^{3}$ of the result. By using the empty skein in $X$ we get a linear functional

$$
Z(K): K_{t}\left(S^{1} \times D^{2}\right) \rightarrow \mathbb{C} .
$$

Let $\kappa(K, c)$ be the value of $Z(K)$ on $S_{c}$. We can then represent $Z(K)$ by

$$
\hat{Z}(K)=\sum_{c} \kappa(K, c) \sigma_{c}
$$

where $\left\{\sigma_{c}\right\}$ is the basis dual to $\left\{S_{c}\right\}$. It is worth noting that the $\kappa(K, c)$ are the colored Kauffman brackets of the knot (which are a version of the colored Jones polynomials of the knot). Indeed, the $c$-th coefficient of the series expansion is 
computed by plugging $S_{c}$ along the core of the regular neighborhood of the knot and evaluating in $S^{3}$, that is by coloring the knot with the $c$-th Jones-Wenzl idempotent and evaluating the result in the skein space of the plane.

Let $\mathcal{F}_{t}(K)$ be the annihilator of $\hat{Z}(K)$ in $K_{t}\left(T^{2} \times I\right)$. This is a left ideal that we call the formal ideal of $K$.

Theorem 2. The ideal $\mathcal{I}_{t}(K)$ lies in $\mathcal{F}_{t}(K)$.

Proof. Recall that a skein $\alpha$ in $T^{2} \times I$ is in $\mathcal{I}_{t}(K)$ if when you glue the 0 -end of $T^{2} \times I$ to $X$, the skein is equivalent to 0 in $K_{t}(X)$. Let $\alpha \in \mathcal{I}_{t}(K)$ and $x \in K_{t}\left(S^{1} \times K^{2}\right)$. Then $\alpha Z(K)$ is a functional on $K_{t}\left(S^{1} \times D^{2}\right)$ and its value on $x$ is computed by embedding the skein $a$ in the knot complement by gluing the cylinder over the torus to the knot complement, and then gluing the solid torus with the skein $x$ in it to the knot complement. But the skein $\alpha$ can be transformed into the zero skein by skein moves taking place entirely in the complement of $x$, hence the value of the functional is zero. Thus the functional itself is zero.

If $\alpha$ is a skein in $K_{t}\left(T^{2} \times I\right)$, then $\alpha$ has a matrix representation coming from the action on the skein module of the solid torus, with basis $\left\{S_{c}\right\}$. The theorem shows that the rows of this matrix are orthogonal to the vector $\hat{Z}(K)$.

For transcendental $t$, is it true that $\mathcal{I}_{t}(K)=\mathcal{F}_{t}(K)$ ?

\section{REFERENCES}

[AL] William Adams, Phillippe Loustaunau, An introduction to Gröbner bases, Graduate Studies in Mathematics, ISSN 1065-7339; 3, AMS, Providence RI, 1994. MR 95g:13025

[B] D. Bullock, Rings of $\mathrm{Sl}_{2}(\mathbb{C})$-characters and the Kauffman bracket skein module, Comment. Math. Helv., 72 (1997), 521-542. MR 2000d:57013

[BH] G. W. Brumfiel, H. M. Hilden, Sl(2) Representations of Finitely Presented Groups, Contemp. Math. 187 (1995). MR 96g:20004

[BP] D. Bullock, J.H. Przytycki, Kauffman bracket skein module quantization of symmetric algebra and so(3), preprint.

[C] P.M. Cohn, Free Rings and their Relations, Academic Press, 1971. MR 51:8155

[CCGLS] D. Cooper, M. Culler, H. Gillett, D.D. Long, P.B. Shalen, Plane Curves associated to character varieties of 3-manifolds, Inventiones Math. 118, pp. 47-84 (1994). MR 95g:57029

[CL] D. Cooper, D. Long, Representation theory and the A-polynomial of a knot, Chaos, Solitons and Fractals, 9 (1998) no 4/5, 749-763. MR 99c:57013

[Co] A. Connes, Noncommutative Geometry, Academic Press, London, 1994. MR 95j:46063

[CS] M. Culler and P.B. Shalen, Varieties of group representations and splittings of 3manifolds, Ann. of Math. 117 (1983), 109-146. MR 84k:57005

[FG] C. Frohman, R. Gelca, Skein Modules and the Noncommutative Torus, Transactions of the AMS, 352 (2000), 4877-4888. MR 2001b:57014

[Ge] R. Gelca, Noncommutative trigonometry and the A-polynomial of the trefoil knot, to appear, Proceedings of Cambridge Philosophical Society.

[HP] J. Hoste, J.H. Przytycki, The $(2, \infty)$-skein module of lens spaces; a generalization of the Jones polynomial, J. Knot Theor. Ramif., 2 (1993), no. 3. 321-333. MR 97f:57019

[K] Ch. Kassel, Quantum Groups, Springer Verlag, 1995. MR 96e:17041

[KM] M. Kapovich, J.J. Millson, On representation varieties of Artin groups, projective arrangements and fundamental groups of smooth complex algebraic varieties, Inst. Hautes Etude Sci. Publ. Math.No. 88 (1998), 5-95. MR 2001d:14024]

[Li] W. B. R. Lickorish, An Introduction to Knot Theory, Springer, GTM 175, 1997. MR 98f:57015

[LM] A. Lubotzky, A. Magid, Varieties of representations of finitely generated groups, Memoirs of the AMS 336 (1985). MR 87e:20021

[PS] J.H. Przytycki, A.S. Sikora, On Skein Algebras and $\mathrm{Sl}_{2}(\mathbb{C})$-Character Varieties, Topology 39 (2000), 115-148. MR 2000g:57026 
[S1] Adam Sikora, A geometric method in the theory of $S L_{n}$-representations of groups, Preprin, xxx.lanl.gov/ math.RT-9806016, (1998).

[Ri1] M. Rieffel, C*-algebras associated with irrational rotations Pac. J. Math. 93, 415-429 (1981). MR 83b:46087

[Ri2] M. Rieffel, Deformation Quantization of Heisenberg Manifolds, Commun. Math. Phys. 122 (1989), 531-562. MR 90e:46060

[Ri3] M. Rieffel, Non-commutative tori - a case study of non-commutative differentiable manifolds, in Geometric and topological invariants of elliptic operators, Proc. AMSIMS-SIAM Jt. Summer Res. Conf., Brunswick/ME (USA) 1988, Contemp. Math. 105, 191-211 (1990). MR 91d:58012

[Ro1] J. Roberts Skeins and Mapping Class Groups, Math. Proc. Camb. Phil. Soc. 115 (1994), 53-77. MR 94m:57035

[Ro2] J. Roberts, Kirby Calculus in Manifolds with Boundary, Turkish J. Math. 21 (1997), 111-117. MR 99c:57045

[We] A. Weinstein, Symplectic groupoids, geometric quantization, and irrational rotation algebras, in Symplectic geometry, groupoids, and integrable systems (Berkeley, CA, 1989), 281-290, Springer, New York, 1991. MR 92g:53028

Department of Mathematics, University of Iowa, Iowa City, Iowa 52242

E-mail address: frohman@math.uiowa.edu

Department of Mathematics and Statistics, Texas Tech University, Lubbock, Texas 79409 and Institute of Mathematics of The Romanian Academy, Bucharest, Romania

E-mail address: rgelca@math.ttu.edu

Department of Mathematics and Computing, University of Wisconsin-Stevens Point, Stevens Point, Wisconsin 54481

E-mail address: Walter.LoFaro@uwsp.edu 